\title{
Effect of preservation of the C-6 spinous process and its paraspinal muscular attachment on the prevention of postoperative axial neck pain in C3-6 laminoplasty
}

\author{
Eiji Mori, MD, Takayoshi Ueta, MD, PhD, Takeshi Maeda, MD, PhD, Itaru Yugué, MD, PhD, \\ Osamu Kawano, MD, PhD, and Keiichiro Shiba, MD, PhD \\ Department of Orthopaedic Surgery, Spinal Injuries Center, lizuka, Fukuoka, Japan
}

\begin{abstract}
OBJECT Axial neck pain after C3-6 laminoplasty has been reported to be significantly lesser than that after C3-7 laminoplasty because of the preservation of the $\mathrm{C}-7$ spinous process and the attachment of nuchal muscles such as the trapezius and rhomboideus minor, which are connected to the scapula. The C- 6 spinous process is the second longest spinous process after that of $\mathrm{C}-7$, and it serves as an attachment point for these muscles. The effect of preserving the C-6 spinous process and its muscular attachment, in addition to preservation of the C-7 spinous process, on the prevention of axial neck pain is not well understood. The purpose of the current study was to clarify whether preservation of the paraspinal muscles of the $\mathrm{C}-6$ spinous process reduces postoperative axial neck pain compared to that after using nonpreservation techniques.
\end{abstract}

METHODS The authors studied 60 patients who underwent C3-6 double-door laminoplasty for the treatment of cervical spondylotic myelopathy or cervical ossification of the posterior longitudinal ligament; the minimum follow-up period was 1 year. Twenty-five patients underwent a C-6 paraspinal muscle preservation technique, and 35 underwent a C-6 nonpreservation technique. A visual analog scale (VAS) and VAS grading (Grades I-IV) were used to assess axial neck pain 1-3 months after surgery and at the final follow-up examination. Axial neck pain was classified as being 1 of 5 types, and its location was divided into 5 areas. The potential correlation between the C-6/C-7 spinous process length ratio and axial neck pain was examined.

RESULTS The mean VAS scores ( \pm SD) for axial neck pain were comparable between the C6-preservation group and the C6-nonpreservation group in both the early and late postoperative stages $(4.1 \pm 3.1$ vs $4.0 \pm 3.2$ and $3.8 \pm 2.9$ vs $3.6 \pm 3.0$, respectively). The distribution of VAS grades was comparable in the 2 groups in both postoperative stages. Stiffness was the most prevalent complaint in both groups (64.0\% and $54.5 \%$, respectively), and the suprascapular region was the most common site in both groups (60.0\% and $57.1 \%$, respectively). The types and locations of axial neck pain were also similar between the groups. The $\mathrm{C}-6 / \mathrm{C}-7$ spinous process length ratios were similar in the groups, and they did not correlate with axial neck pain. The reductions of range of motion and changes in sagittal alignment after surgery were also similar.

CONCLUSIONS The C-6 paraspinal muscle preservation technique was not superior to the C6-nonpreservation technique for preventing postoperative axial neck pain.

http://thejns.org/doi/abs/10.3171/2014.11.SPINE131153

KEY WORDS laminoplasty; axial neck pain; paraspinal muscle; cervical spine; spinous process; trapezius muscle

$\mathrm{L}$ AMINOPLASTY, which evolved from extensive laminectomy in the treatment of cervical spinal cord compression, is an established posterior cervical decompression procedure for multisegmental cervical myelopathy. ${ }^{5,9,20}$ Although acceptable neurological improvement after laminoplasty is achieved, postoperative complications or problems such as axial neck pain, reduction of cervical range of motion (ROM), and changes in spinal alignment are often induced..$^{1,3,7,12}$ Axial neck pain, typified by persistent pain around the neck and shoulder, is a notorious postoperative complication after laminoplasty. ${ }^{8,12,18}$ The incidence of axial neck pain has been reported

ABBREVIATIONS CSM = cervical spondylotic myelopathy; JOA = Japanese Orthopaedic Association; OPLL = ossification of the posterior longitudinal ligament; ROM = range of motion; VAS = visual analog scale.

SUBMITTED December 16, 2013. ACCEPTED November 4, 2014.

INCLUDE WHEN CITING Published online December 19, 2014; DOI: 10.3171/2014.11.SPINE131153.

DISCLOSURE The authors report no conflict of interest concerning the materials or methods used in this study or the findings specified in this paper. 
to range from $5 \%$ to $80 \% .6,13,18$ Several techniques have been developed by surgeons who attempted to reduce axial neck pain after laminoplasty. ${ }^{14,17,21}$ In a comparative study between C3-6 and C3-7 laminoplasty, Hosono et al. ${ }^{6}$ reported that postoperative axial neck pain was significantly less severe after C3-6 laminoplasty than after C3-7 laminoplasty. They suggested that preservation of the $\mathrm{C}-7$ spinous process and the origin of the trapezius and rhomboid minor muscles on the C-7 spinous process was key to preventing postoperative axial neck pain. The C-7 spinous process is an important muscular attachment point for the trapezius and rhomboid minor muscles connecting to the scapula. They hypothesized that disruption of the musculotendinous connection between the C-7 spinous process and the scapula was one of the causes of postoperative axial neck pain. The $\mathrm{C}-6$ spinous process is the second longest spinous process after the $\mathrm{C}-7$ process, and it also serves as an attachment point for the rhomboid minor and the speculum rhomboideum section of the trapezius, which is composed of the middle fibers of the trapezius and contains a very strong tendinous component. ${ }^{16}$ Therefore, we postulated that preservation of the paraspinal muscles of the C-6 spinous process in addition to the $\mathrm{C}-7$ spinous process might decrease postoperative axial neck pain. The relationship between preservation of the paraspinal muscles of the C-6 spinous process and axial neck pain is not well understood. The purpose of the current study was to clarify whether preservation of the paraspinal muscles of the C-6 spinous process reduces postoperative axial neck pain.

\section{Methods}

\section{Patient Population}

In total, 81 patients with no history of cervical surgery underwent a C3-6 spinous process-splitting double-door laminoplasty for the treatment of cervical spondylotic myelopathy (CSM) or cervical ossification of the posterior longitudinal ligament (OPLL) between January 2009 and December 2010. Thirty-three patients were treated with a paraspinal muscle preservation technique at the C-6 level (C6-preservation group), and 48 were treated with a paraspinal muscle nonpreservation technique at the C-6 level (C6-nonpreservation group). A surgeon (I.Y.) who was interested in preservation techniques performed the C6preservation laminoplasties, and others (T.U. and T.M.) performed the C6-nonpreservation laminoplasties, our conventional technique. Although selection of the surgeon and the patients was not randomized in the current study, the patients were not informed about the C- 6 spinous process preservation procedure. Of the patients, 60 were followed up and examined for more than 1 year. The ethics committee of our institution approved the study. Of the 60 patients, 44 were men, and the mean age of the group was 66.5 years (range $47-81$ years). Forty-three patients had CSM and 17 had cervical OPLL. The mean follow-up durations were 3 years 1 month (range 1.8-3.8 years) in the C6-preservation group and 2 years 10 months (range 1.5-3.8 years) in the C6-nonpreservation group. Twentyfive patients underwent C6-preservation laminoplasty, and 35 underwent C6-nonpreservation laminoplasty. No significant differences in age, sex, or diagnosis were observed between the 2 groups (Table 1 ).

\section{Surgical Procedures}

In both of the groups, a midline skin incision was made from the $\mathrm{C}-2$ spinous process to the $\mathrm{C}-7$ spinous process. The nuchal ligament was incised in the midline. Then, the incision was continued between the bilateral splenius capitis and semispinalis capitis down to the spinous processes from C-3 to C-6. All the bilateral muscles attached to the C-2 and C-7 spinous processes were preserved by the procedures performed in both groups.

In the C6-preservation procedure, the paraspinal muscles were dissected, detached from the C-3 to C-5 posterior aspect while the attachment points of the paraspinal muscles such as rotators, multifidus, semispinalis cervicis, rhomboid minor, and the speculum rhomboideum of the trapezius (if attached to the spinous processes of C-6) were left intact, and retracted to ensure exposure of the laminae and the medial border of the facet joints. The epidural space was exposed after opening the $\mathrm{C}-3$ split spinous process using a surgical bur, with lateral gutters at the medial border of the facet joint bilaterally. After midline blunt dissection with cutting of the interspinous process ligament between the C-6 and C-7 spinous processes, the epidural space was minimally exposed. The attachments of the paraspinal muscles to the C-6 and C-7 spinous processes were left intact. The C-4, C-5, and C-6 spinous processes were split longitudinally by a thread saw passed through the epidural space from C-3 to C-6/C-7, and lateral gutters were made bilaterally. At the C-6 level, bilateral gutters were made after minimal exposure of the medial border of the facet joint by retracting the paraspinal muscles from the lamina with a Penfield elevator while leaving the muscular attachment to the spinous process undisturbed. Bilateral halves of split spinous processes and laminae were lifted and bilaterally opened. A ceramic spacer was placed between the split spinous processes at each level (Fig. 1).

In the C6-nonpreservation procedure, the paraspinal muscles were dissected and detached thoroughly from the C-3 to C-6 posterior aspect. Longitudinal splitting of the spinous process from C-3 to C- 6 was performed with a thread saw passed through the epidural space from $\mathrm{C}-6 / \mathrm{C}-7$ to $\mathrm{C}-2 / \mathrm{C}-3$. The procedures for opening the split

TABLE 1. Patient characteristics and surgical invasion

\begin{tabular}{lccc}
\hline \multicolumn{1}{c}{ Characteristic } & $\begin{array}{c}\text { C6-Preservation } \\
\text { Group }(\mathrm{n}=25)\end{array}$ & $\begin{array}{c}\text { C6-Nonpreservation } \\
\text { Group }(\mathrm{n}=35)\end{array}$ & p Value \\
\hline $\begin{array}{c}\text { Mean age in yrs } \\
\text { (range) }\end{array}$ & $66.1(47-79)$ & $66.7(48-81)$ & $\mathrm{NS}$ \\
\hline $\begin{array}{c}\text { Sex (male/female) } \\
\mathrm{CSM}^{*}\end{array}$ & $19: 6$ & $25: 10$ & $\mathrm{NS}$ \\
\hline $\mathrm{OPLL}^{*}$ & 17 & 26 & $\mathrm{NS}$ \\
\hline $\begin{array}{c}\text { Mean op time } \\
\text { (mins) } \dagger\end{array}$ & 8 & 9 & \\
\hline mean EBL $(\mathrm{g}) \dagger$ & $60.2 \pm 41.7$ & $40.6 \pm 32.0$ & $<0.05$ \\
\hline
\end{tabular}

$\mathrm{EBL}=$ estimated blood loss; NS = not significant.

* Values are the number of patients.

$\dagger$ Values are mean \pm SD. 

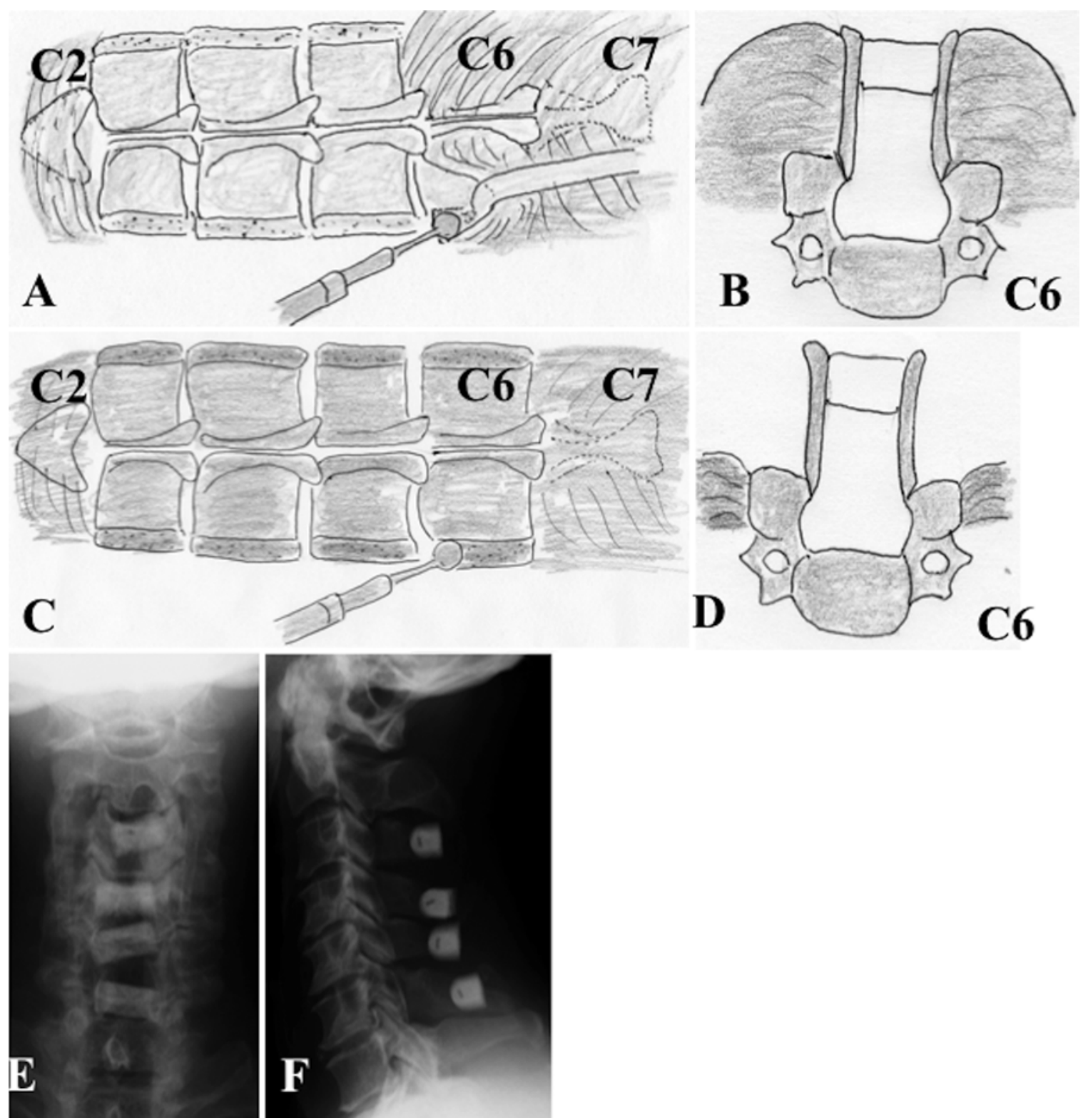

FIG. 1. A and B: In the C6-preservation procedure, the paraspinal muscles were dissected and detached from the C3-5 posterior aspects. The C3-6 spinous processes were cut longitudinally at the midline using a thread saw, and the attachments of the paraspinal muscles, such as rotators, multifidus, semispinalis cervicis, rhomboid minor, and the speculum rhomboideum of the trapezius, if it was attached, to the spinous processes of C-6 were left intact. At the C6 level, bilateral gutters were made after minimal exposure of the medial border of the facet joint by retracting the paraspinal muscles from the lamina with a Penfield elevator while leaving the muscular attachment to the spinous process undisturbed. $\mathbf{C}$ and $\mathbf{D}$ : In the C6-nonpreservation procedure, the paraspinal muscles were dissected and detached thoroughly from the C3-5 and C-6 posterior aspects. E and F: Anteroposterior and lateral radiographs after the C6-preservation procedure. Copyright (A-D) Eiji Mori. Published with permission.

spinous processes and placement of ceramic spacers were the same as those described for the C6-preservation procedure (Fig. 1).

Each patient in both groups wore a cervical orthosis for 10 days after surgery.

\section{Clinical Examination}

A 10-point visual analog scale (VAS) and VAS grading were used to assess axial neck pain 1-3 months after surgery and at the final follow-up observation. Pain around the neck and shoulder persisting for more than 1 week in the 1-3 months after surgery or for more than 1 month after surgery was considered postoperative axial neck pain. Patients were asked to mark their level of pain under the condition of no analgesics. The VAS grades were defined as follows: Grade I, 0-2.5 points; Grade II, 2.6-5.0 points; Grade III, 5.1-7.5 points; and Grade IV, 7.6-10.0 points. Axial neck pain was classified as 1 of 5 types as follows: pain, stiffness, tension, tightness, or traction. The axial neck pain areas of distribution were defined as follows: nuchal region, suprascapular region, superior angle of the scapular region, scapular region, and interscapular region (Fig. 2). 
The type(s) and area(s) of distribution of axial neck pain (patients were allowed to select multiple types and/or areas) were examined and defined as positive when patients experienced pain 1-3 months after surgery or at the final follow-up observation. The examination for axial neck pain was performed by a clerical nonsurgical staff member who was blinded to the patients' surgical status. The correlation between the ratio of the length of the $\mathrm{C}-6$ spinous process to that of the $\mathrm{C}-7$ spinous process $(\mathrm{C}-6 / \mathrm{C}-7$ spinous process length ratio) and the VAS score for axial neck pain was examined after surgery or at the final follow-up. These items concerning axial neck pain were compared between the C6-preservation and C6-nonpreservation groups. Surgical invasion was also compared between the groups. The $\mathrm{ROM}$ and sagittal alignment from C-2 to C-7 were examined before and after surgery and compared between the 2 groups. Neurological function was assessed by using the Japanese Orthopaedic Association (JOA) scale (full score 17), which was administered by a doctor who was blinded to the patients' surgical status.

\section{Statistical Analysis}

The clinical parameters of the 2 groups were compared using the Mann-Whitney U-test and the Fisher exact test. The correlation between the $\mathrm{C}-6 / \mathrm{C}-7$ spinous process length ratio of each patient and his or her VAS score for axial pain was analyzed using Pearson's correlation coefficient. Statistical analysis was performed using the JMP program (version 8; SAS Institute Japan). p values of < 0.05 were considered statistically significant. Means are presented \pm SD.

\section{Results}

The mean surgical time was significantly longer and the estimated blood loss was significantly greater in the C6-preservation group than in the C6-nonpreservation group (Table 1).

\section{VAS Scores for Axial Neck Pain and VAS Grades}

The mean VAS score for axial neck pain in the C6preservation group was equivalent to that in the C6nonpreservation group 1-3 months after surgery (4.1 \pm 3.1 vs $4.0 \pm 3.2$, respectively). Moreover, the mean VAS scores for axial neck pain were comparable between the 2 groups at the final follow-up observation $(3.8 \pm 2.9$ vs $3.6 \pm 3.0$, respectively) (Fig. 3). The VAS grade distributions were comparable between the groups at both 1-3 months after surgery and the final follow-up observation (Fig. 4).

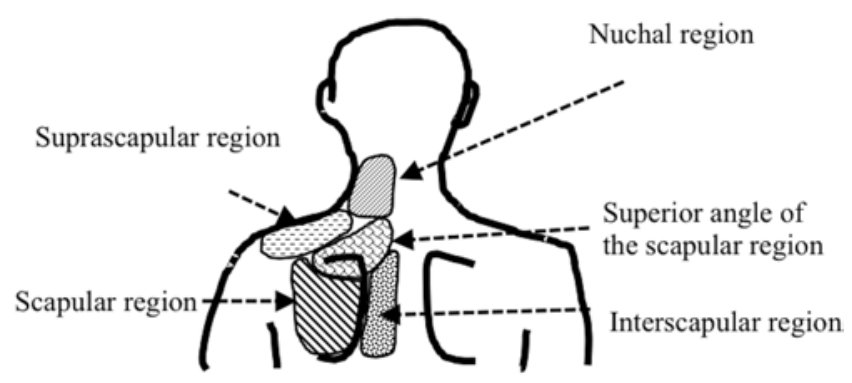

FIG. 2. Locations of axial neck pain (5 separate regions).

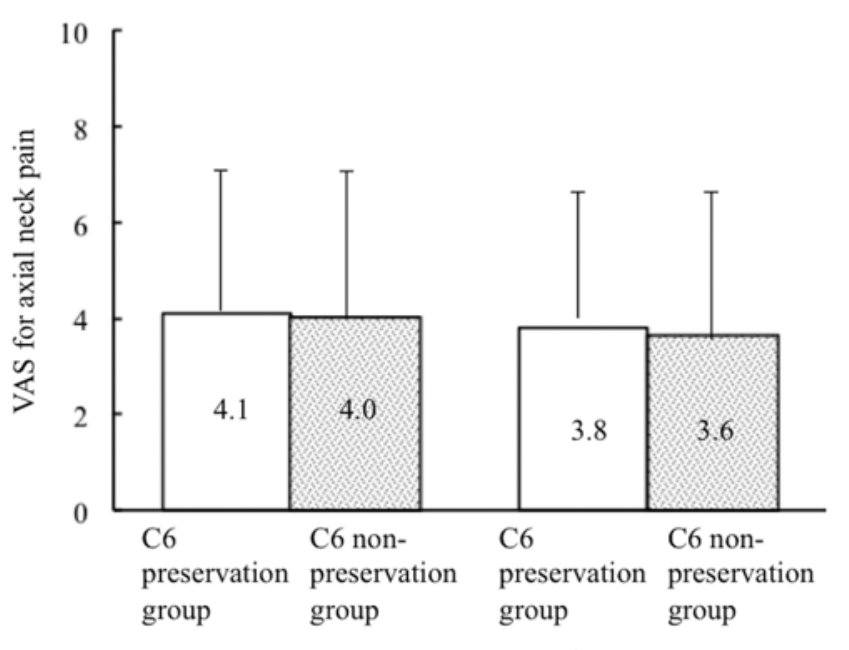

FIG. 3. VAS scores for axial neck pain in the C6-preservation and C6nonpreservation groups 1-3 months after surgery and at the final followup observation. The white columns denote the C6-preservation group, and the gray columns denote the C6-nonpreservation group. There were no differences in the mean VAS scores of the 2 groups at either postoperative stage.

\section{Type of Axial Neck Pain}

Among the types of axial neck pain, stiffness was the most prevalent complaint in both the C6-preservation and C6-nonpreservation groups $(64.0 \%$ and $54.5 \%$, respectively). Subsequently, pain was prevalent in both groups ( $40.0 \%$ and $39.4 \%$, respectively). The prevalences of the types of axial neck pain were comparable between the groups for all 5 types (Fig. 5).

\section{Location of Axial Neck Pain}

Among the locations of axial neck pain, the suprascapular region was the most common site in both the C6preservation and C6-nonpreservation groups $(60.0 \%$ and $57.1 \%$, respectively). Subsequently, the nuchal region was commonly affected in both groups $(48.0 \%$ and $45.7 \%$, respectively). The prevalences of axial neck pain were comparable between the groups at all 5 regions (Table 2).

\section{C-6/C-7 Spinous Process Length Ratio and Axial Neck Pain}

The mean C-6/C-7 spinous process length ratios were comparable between the C6-preservation and C6nonpreservation groups $(76.9 \pm 9.9$ vs $78.0 \pm 11.2$, respectively). The C-6/C-7 spinous process length ratios of the patients were not significantly correlated with the VAS scores for axial neck pain either 1-3 months after surgery or at the final follow-up in the C6-preservation group (Fig. 6). Similarly, no significant correlation between the C-6/C-7 spinous process length ratios and the VAS scores for axial neck pain was observed for either postoperative stage in the C6-nonpreservation group (Fig. 7).

\section{Radiographic and Neurological Outcomes}

The reductions of ROM at the final follow-up visit, which were greater in the subgroup of patients with OPLL than in 


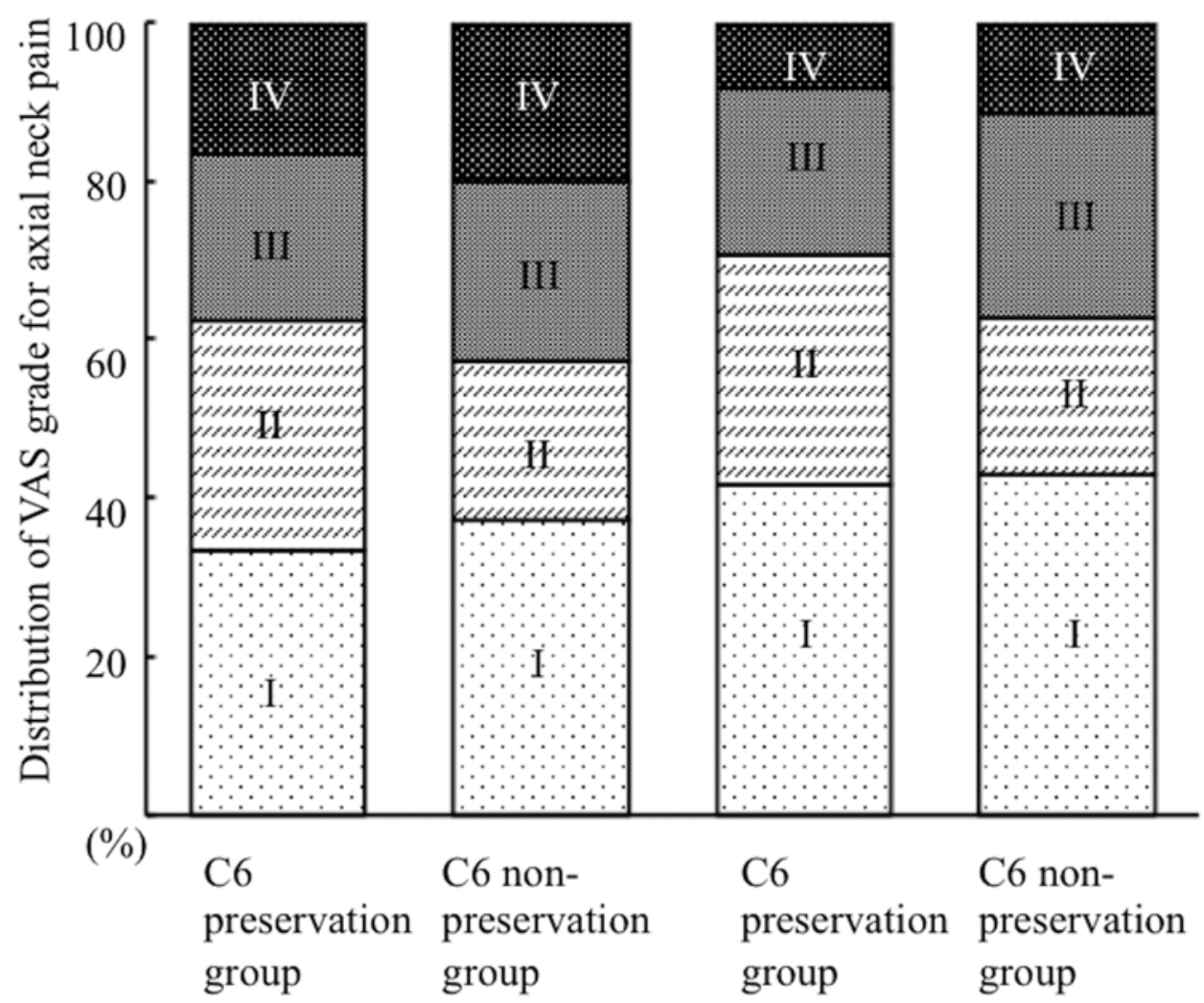

\section{Postoperative months 1-3 Final follow-up observation}

FIG. 4. The distribution of VAS grades for axial neck pain in the C6-preservation and C6-nonpreservation groups 1-3 months after surgery and at the final follow-up observation. There were no differences in the distributions of VAS grades between the 2 groups for either postoperative stage.

the subgroup of patients with CSM, were comparable between the C6-preservation and C6-nonpreservation groups in both of these subgroups (Table 3). Neither fracture of the lamina nor dislodgement of a spacer was observed in either of the 2 groups in the follow-up period. The sagittal alignment conversion of preoperative lordosis to postoperative kyphosis was observed in only 2 patients in the C6nonpreservation group. A loss of C2-7 lordosis of $>10^{\circ}$ was observed in 2 patients $(8.0 \%)$ in the C6-preservation group and in 3 patients (8.6\%) in the C6-nonpreservation group. The JOA score and its recovery rate were not significantly different between the 2 groups (Table 4). The mean hospital stay was $43.7 \pm 21.3$ days in the C6-preservation group and $42.5 \pm 16.0$ days in the C6-nonpreservation group. The patients participated in a rehabilitation program after surgery during their stay in the hospital. Therefore, they were not discharged soon after their surgery. In the C6preservation group, 3 patients (12\%) experienced dysesthesia in the upper extremities after surgery. These unpleasant symptoms were relieved in a few weeks with conservative treatment. One patient had cerebrospinal fluid pooling in the surgical site, which was identified after surgery and was associated with headache and nausea. He was treated conservatively and successfully without neurological worsening. Another patient had night delirium that lasted only a few nights. In the C6-nonpreservation group, there were 5 patients (14.3\%) who experienced postsurgical dysesthesia in the upper extremities, which was relieved with conservative treatment in a few weeks. One patient was diagnosed with pneumonia after surgery and was treated successfully with antibiotics. One patient with an electrolyte abnormality and the patient with night delirium were managed conservatively. It was fortunate that neither neurological worsening (including C-5 paresis) nor any other serious complications, such as surgical site infection, nerve injury, or hematoma, occurred in either group.

\section{Discussion}

Postoperative axial neck pain typified by persistent pain around the neck and shoulders is a common problem associated with cervical laminoplasty. $7,8,12,18$ There have been wide variations in the incidence of axial neck pain after various types of laminoplasty techniques. ${ }^{6,13,18}$ Several posterior cervical decompression techniques, such as skip laminectomy, ${ }^{21}$ segmental partial laminectomy, ${ }^{17}$ and muscle-preserving laminoplasty, ${ }^{14}$ have been introduced in an attempt to reduce postoperative complications, including axial neck pain. In terms of preventing axial neck pain, the results were not always favorable. ${ }^{22,23}$ Although the exact cause of axial neck pain has not been detected, intraoperative invasion to the nuchal muscles is presumed 


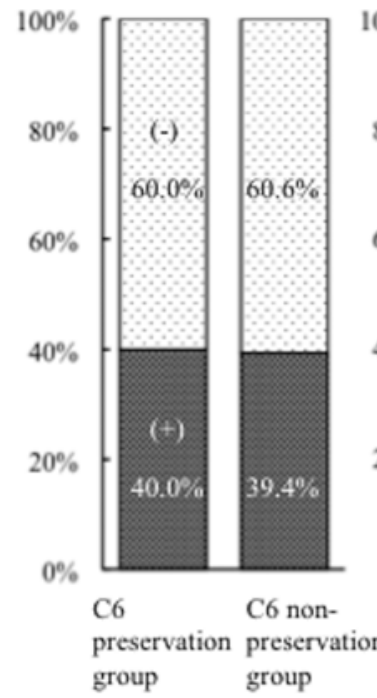

[pain ]

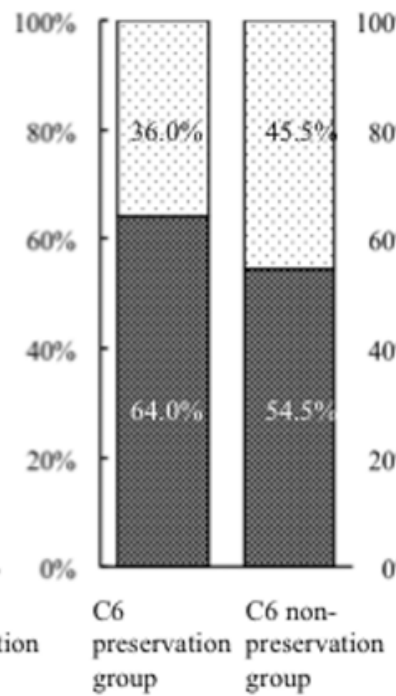

[ stiffness ]

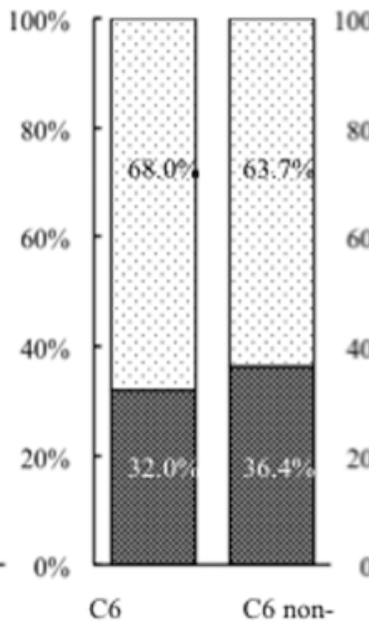

preservation preservation group group

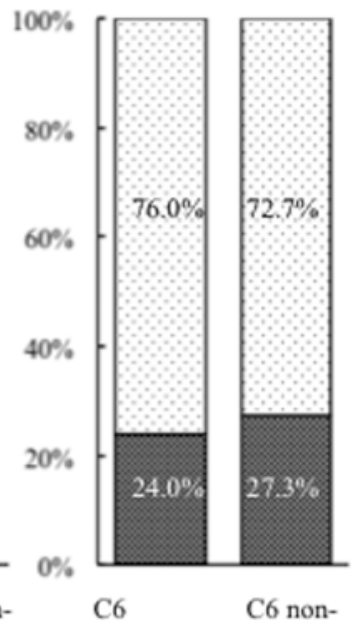

preservation preservation group group

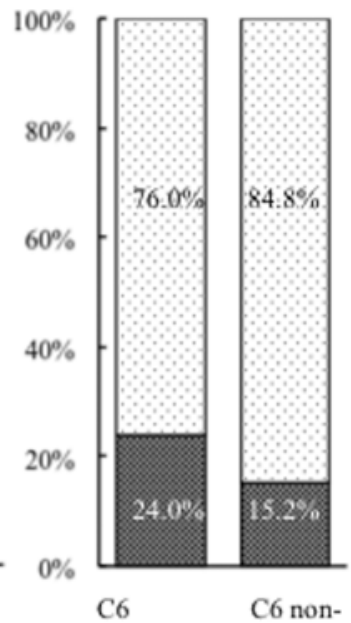

preservation preservation group group

$$
\text { [ tension ] [ tightness ] }
$$

[ traction ]

FIG. 5. Types of axial neck pain. The dark columns denote positivity, and the spotted columns denote negativity concerning the type of pain. Stiffness was the most common complaint in both groups, and pain was the second most prevalent complaint. The prevalences of axial neck pain were comparable between the 2 groups for all 5 types.

to be involved in this complication..$^{14,17,21}$ Nuchal muscles, such as the trapezius, rhomboid minor, splenius capitis, and serratus posterior superior muscles, are more densely and closely bound to the C-7 spinous process than other upper or middle cervical vertebrae. ${ }^{11}$ Moreover, the maximum force generated by the trapezius has been reported among the cervical vertebrae to be the greatest at C-7. ${ }^{10}$ In clinical research, the authors of one study reported that axial pain was induced after upper thoracic surgery and required detachment and spreading of the spinous process or vertebral arch at the cervicothoracic junction; these results were in contrast to those after middle or lower thoracic surgery, which was associated with no strong postoperative pain around the surgical wound. ${ }^{19}$ These findings suggest that the connection of the nuchal muscles with the C-7 spinous process is very important and related to the pathology of postoperative axial neck pain. Focusing on preserving the large spinous process of $\mathrm{C}-7$ as the important origin of the nuchal muscles, Hosono et al. ${ }^{6}$ conducted a comparative study of conventional C3-7 laminoplasty and C3-6 laminoplasty by limiting the range of decompression with preservation of the $\mathrm{C} 7$ spinous process. They

TABLE 2. Prevalence of axial neck pain at each of the 5 locations*

\begin{tabular}{lccc}
\hline \multicolumn{1}{c}{ Region } & $\begin{array}{c}\text { C6-Preservation } \\
\text { Group }(\mathrm{n}=25)\end{array}$ & $\begin{array}{c}\text { C6-Nonpreservation } \\
\text { Group }(\mathrm{n}=35)\end{array}$ & p Value \\
\hline Nuchal & $12(48.0)$ & $16(45.7)$ & NS \\
\hline Suprascapular & $15(60.0)$ & $20(57.1)$ & NS \\
\hline $\begin{array}{l}\text { Superior angle of } \\
\text { the scapula }\end{array}$ & $4(16.0)$ & $7(20.0)$ & NS \\
\hline Scapular & $1(4.0)$ & $3(8.6)$ & NS \\
\hline Interscapular & $1(4.0)$ & $4(11.4)$ & NS \\
\hline
\end{tabular}

* Values represent number (\%) of patients. demonstrated the superiority of C3-6 laminoplasty over C3-7 laminoplasty in regard to axial neck pain; the incidence of postoperative axial neck pain was only $5.4 \%$ after C3-6 laminoplasty versus $29 \%$ after the $\mathrm{C} 3-7$ procedure. They emphasized the importance of preserving the C--7

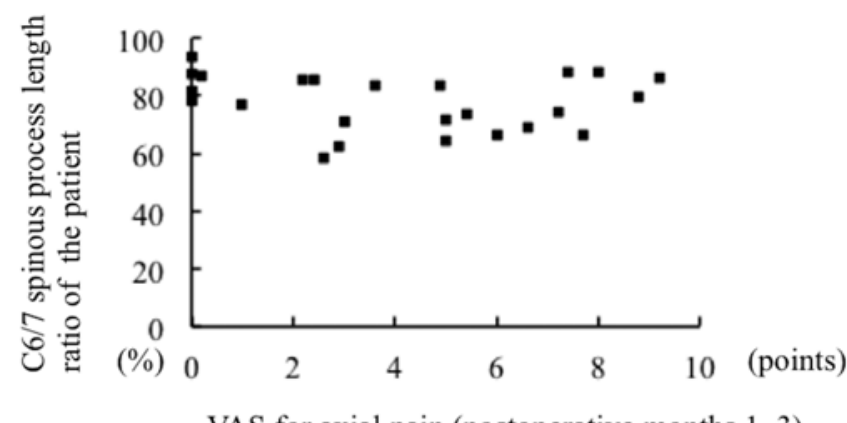

VAS for axial pain (postoperative months 1-3)

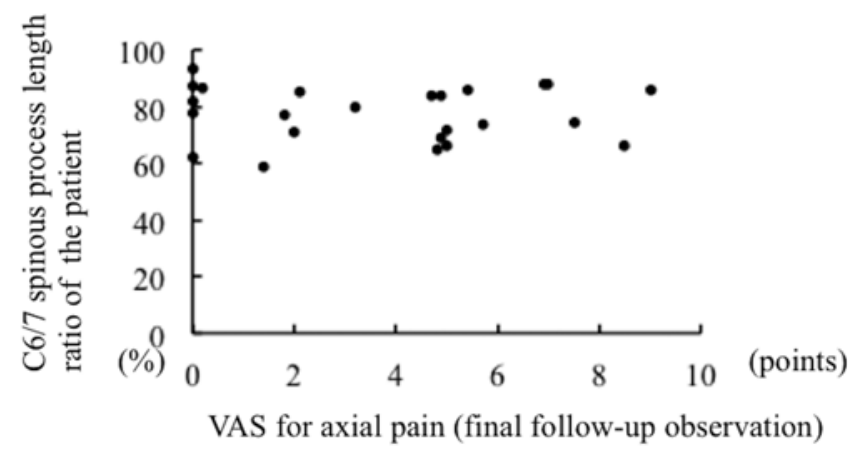

FIG. 6. Correlation between the C-6/C-7 spinous process length ratios of the patients and the VAS scores for axial neck pain in the C6preservation group. The C-6/C-7 spinous process length ratios were not significantly correlated with the VAS scores for axial neck pain either 1-3 months after surgery (upper) or at the final follow-up observation (lower). 


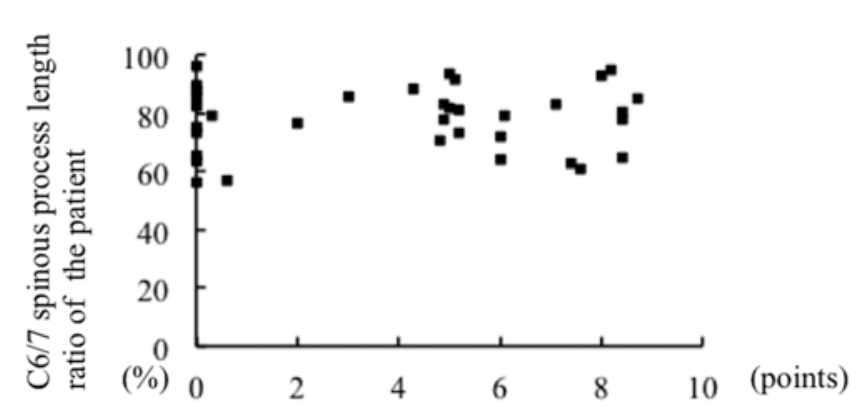

VAS for axial pain (postoperative months 1-3)

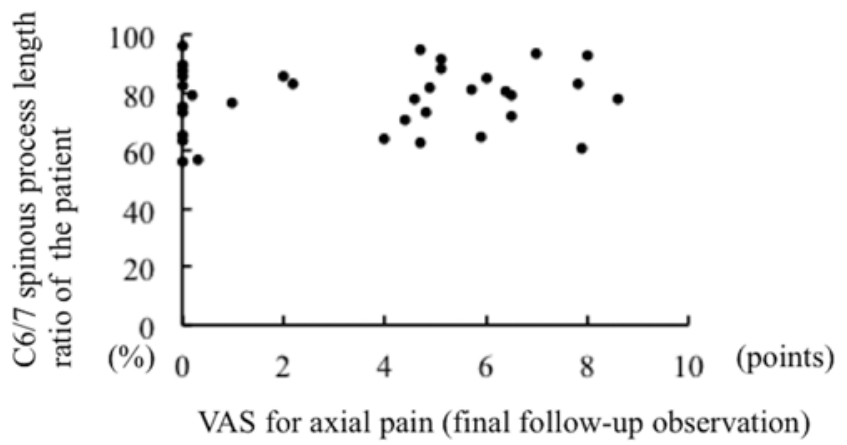

FIG. 7. Correlation between the C-6/C-7 spinous process length ratios and the VAS scores for axial neck pain in the C6-nonpreservation group. The C-6/C-7 spinous process length ratios were not significantly correlated with the VAS scores for axial neck pain either 1-3 months after surgery (upper) or at the final follow-up observation (lower).

spinous process and the origin of the trapezius and rhomboid minor muscles on the C-7 spinous process for preventing postoperative axial neck pain. Patients with axial neck pain often experience increasing pain in the sitting or upright position, in contrast to decreasing pain in a lying position. Hosono et al. ${ }^{6}$ provided the following explanation for the pain pattern. Downward displacement of the upper extremities occurs in the sitting position because of gravity. The trapezius and rhomboid minor muscles connecting the C-7 spinous process and the scapula are stretched by adduction of the scapula with downward displacement of the upper extremities. The disruption of the connection at the C-7 spinous process, serving as the important muscu-

TABLE 3. ROM before and after surgery

\begin{tabular}{|c|c|c|c|}
\hline $\begin{array}{l}\text { Condition and } \\
\text { Parameters }\end{array}$ & $\begin{array}{c}\text { C6 Preservation } \\
\text { Group }(n=25)\end{array}$ & $\begin{array}{l}\text { C6 Nonpreservation } \\
\text { Group }(n=35)\end{array}$ & $\begin{array}{c}\mathrm{p} \\
\text { Value }\end{array}$ \\
\hline No. of patients w/ CSM & 17 & 26 & \\
\hline Preop ROM $\left({ }^{\circ}\right)^{*}$ & $35.2 \pm 11.7$ & $30.1 \pm 13.8$ & NS \\
\hline Final FU ROM $\left({ }^{\circ}\right)^{*}$ & $28.8 \pm 11.2$ & $25.8 \pm 11.5$ & NS \\
\hline$\%$ ROM† & 81.8 & 85.7 & NS \\
\hline No. of patients w/ OPLL & 8 & 9 & \\
\hline Preop ROM $\left({ }^{\circ}\right)^{*}$ & $23.5 \pm 9.0$ & $21.1 \pm 13.4$ & NS \\
\hline Final FU ROM $\left({ }^{\circ}\right)^{*}$ & $18.5 \pm 9.7$ & $14.8 \pm 7.7$ & NS \\
\hline$\%$ ROM (\%)† & 78.7 & 70.1 & NS \\
\hline
\end{tabular}

TABLE 4. Neurological outcome

\begin{tabular}{lccc}
\hline & \multicolumn{2}{c}{ Mean \pm SD } & \\
\cline { 2 - 3 } Outcome & $\begin{array}{c}\text { C6-Preservation } \\
\text { Group }(\mathrm{n}=25)\end{array}$ & $\begin{array}{c}\text { C6-Nonpreservation } \\
\text { Group }(\mathrm{n}=35)\end{array}$ & p Value \\
\hline Preop JOA score & $10.2 \pm 2.9$ & $9.8 \pm 2.7$ & NS \\
\hline Final FU JOA score & $14.1 \pm 3.1$ & $13.6 \pm 3.0$ & NS \\
\hline Recovery rate $(\%)^{*}$ & $60.8 \pm 21.3$ & $57.8 \pm 29.5$ & NS \\
\hline${ }^{*}$ Recovery rate calculated as follows: (JOA at final follow-up - preop JOA/17 \\
- preop JOA) $\times 100$.
\end{tabular}

lar attachment point for these muscles, might increase the stress on the remaining muscles attached to other spinous processes, resulting in persistent axial pain.

The attachment of the trapezius ranges widely from the occipital bone to the T-12 spinous process. In particular, the speculum rhomboideum section, which is composed of the middle fibers of the trapezius, is very strong because of the presence of a very strong tendinous component. From an anatomical study using 50 cadavers, Ono et al. ${ }^{16}$ reported that the speculum rhomboideus of the trapezius attached to the spinous process between C-3 and T-3, and its center was located at the C-7 spinous process in more than half of the individuals. The attachment of the rhomboid minor muscle to the spinous process occurred between C-5 and C-7. The C-6 spinous process is the second longest spinous process next to the $\mathrm{C} 7$ in the cervical region, and it plays a role as an attachment point for these muscles. Ono et al. ${ }^{16}$ also stated that $>50 \%$ preservation of the attachment of the speculum rhomboideum of the trapezius was possible in $72 \%$ of the patients who underwent C3-6 laminoplasty with preservation of the C-7 spinous process and in $88 \%$ of the patients who underwent C3C5 laminoplasty. The rhomboid minor muscle was possibly spared without complete dissection of the muscular attachment at the spinous process in $35 \%$ of the patients who underwent C3-6 laminoplasty and in $>50 \%$ of the patients who underwent C3-5 laminoplasty. Therefore, preserving the attachment of the paraspinal muscles to the C-6 spinous process in addition to the $\mathrm{C}-7$ spinous process increases the preservation of the muscular attachment at the spinous process in the central area of the speculum rhomboideus of the trapezius and the rhomboid minor, potentially reducing axial neck pain more than that after using the preservation technique on the C-7 spinous process alone. However, the relationship between preservation of the C-6 spinous process and axial neck pain has not been studied thoroughly. Contrary to our expectations, the mean VAS scores and the distributions of VAS grade for axial neck pain were amazingly comparable between the C6-preservation and C6-nonpreservation groups in both the early and late postoperative stages. In addition, neck pain as assessed by VAS scores and the type of pain and its location were similar between the 2 groups. These results demonstrate that our paraspinal muscle preservation procedure for the C-6 spinous process was not superior to the C6-nonpreservation technique in terms of reducing postoperative axial neck pain.

We assume that the reason that the C6-preservation procedure did not achieve a successful result regarding 
axial neck pain includes anatomical and technical aspects. We did not know the range of attachment of the speculum rhomboideum of the trapezius and the rhomboid minor to the spinous process in each patient; therefore, we did not understand the extent of preservation of these muscles with regard to treatment of the paraspinal muscles at the C- 6 spinous process. The maximum degree of preservation of these muscles and the resulting effect on the control of axial neck pain might be reached with preservation of the C-7 spinous process regardless of the C- 6 procedure. Although we also examined the relationship between the length of the C6 spinous process and axial neck pain, the C-6/C-7 spinous process length ratios were similar in the 2 groups, and furthermore, they did not correlate with axial neck pain. From a technical viewpoint, our preservation technique for paraspinal muscles at the C-6 spinous process did not clarify whether the $\mathrm{C}-6$ spinous process and its attachment of paraspinal muscles were left intact. The longitudinally split C-6 spinous process and lamina were spread similar to opening a double door while leaving the muscular attachment to the split spinous processes undisturbed, but the connections of the spinous process to the paraspinal muscles and nuchal ligament were different than those in the original alignment. Our preservation technique at the C-6 level might be insufficient for preventing axial neck pain, but another decompression technique allowing the C- 6 spinous process and its paraspinal muscle attachment to be left intact could be successful.

Stiffness and tension, which were frequently observed in the patients in our study, seem to be similar to symptoms derived from back muscle diseases after lumbar surgery. ${ }^{15}$ Almost half of the patients in the present study presented with pain in the nuchal or suprascapular region, and these areas seem to correspond to those in which nuchal muscles are stretched between the scapula and spinous processes by adduction of the scapula with downward displacement of the upper extremities. These findings suggest that the pathology of axial neck pain is mainly attributable to intraoperative invasion of the nuchal muscles.

The pathology of neck pain after posterior cervical surgery is multifactorial. Highsmith et al. ${ }^{4}$ conducted a comparative study of laminoplasty and laminectomy with fusion in patients with cervical stenotic myelopathy. Preoperative neck pain VAS scores of 3.2 increased slightly postoperatively to 3.4 in the laminoplasty group, whereas in the fusion group, VAS scores significantly improved from 5.8 to 3.0. The authors concluded that cervical fusion significantly reduces neck pain in patients with cervical stenotic myelopathy. Motion-related pain, which is improved by stabilization with fusion, is an important factor in neck pain. This clinical study demonstrated this kind of neck pain. The pathology of preoperative neck pain is possibly different from that of postoperative neck pain. Although pain was reduced after fusion, postoperative neck pain in the fusion group was comparable to that in the laminoplasty group. Spinous processes and paraspinal muscular attachments were not preserved in either of the groups. Postoperative neck pain might have been related to the resection of spinous processes associated with the dissection and detachment of paraspinal muscles.

We used only pain characteristics such as intensity, pat- tern, and location to evaluate axial neck pain. We believe that our assessment for neck pain was not sufficient, because neck pain is an individual unpleasant sensory and emotional experience that is affected by various factors, including cervical pathologies and psychological issues. We found no differences in the VAS scores for axial neck pain between the CSM and OPLL subgroups 1-3 months after surgery or at the final follow-up observation (3.8 \pm 3.2 vs $4.7 \pm 3.0[p>0.05]$ and $3.5 \pm 3.1$ vs $4.0 \pm 2.5[p>$ $0.05]$, respectively). We should have adopted the use of additional instruments such as the Neck Disability Index, ${ }^{24}$ which is most widely used to assess the effect of neck pain on the ability to manage everyday tasks, and the Selfrating Depression Scale ${ }^{2}$ which is used for the assessment of psychological factors. These additional evaluations probably would have helped us identify the differences in axial neck pain in our 2 groups of patients.

When VAS Grade III and IV axial neck pain $(>5.0$ VAS points) in the present study was considered nearly comparable to moderate and severe axial pain (medicine or physical therapy for the painful muscles regularly needed), respectively, the incidence of axial neck pain in the late postoperative period was $34 \%$ in our study compared with $5.4 \%$ in the study of Hosono et al., ${ }^{6}$ in which these grades of pain were defined as axial neck pain. Although it is not precise to compare the results directly between these 2 studies because of the differences in axial neck pain measurements, the incidence of postoperative axial neck pain was higher in our study than in the study of Hosono et al. despite the use of C3-6 laminoplasty with C-7 spinous process preservation in both studies. This finding suggests that the difference in the laminoplasty techniques, doubledoor laminoplasty in our study and open-door laminoplasty in their study, possibly contributes to the pathology of axial neck pain. The purpose of the present study was to examine the efficacy of preserving the paraspinal muscular attachment to the C- 6 spinous process in reducing postoperative axial pain. Our technique for preserving the C-6 spinous process did not effectively diminish pain.

The current study has some limitations. The study was retrospective, and the patients were not randomly assigned to their treatment group. Not only the individual surgeons but also the number of surgeons between the 2 groups were different. There are biases based on the technical variety of the surgeons and patient selection. A randomized prospective study without any biases would have been ideal. However, this was a preliminary nonrandomized comparative study aimed at investigating the advantages of the paraspinal muscle preservation technique before a randomized study is conducted. Each surgeon in each group was an expert spine surgeon with more than 10 years of experience who had no technical difficulty in handling the paraspinal muscles. Therefore, the difference in the surgeons between our 2 groups does not seem to highlight a difference in the surgeons' skills. The sample size was small, and it included a combined population of patients with CSM and cervical OPLL. There was no image assessment of the paraspinal muscles at the C-6 level, including atrophy or signal changes of muscles concomitant with postoperative clinical evaluations. A prospective randomized study with a large sample size would be re- 
quired to compare the effect of differences in axial neck pain on the clinical outcome of C3-6 laminoplasty versus that of C3-5 laminoplasty with the preservation of the C-6 spinous process out of the range of decompression.

\section{Conclusions}

The VAS scores for axial neck pain and the distribution of VAS grades in both the early and late postoperative stages and the types and locations of axial neck pain were comparable between the C-6 paraspinal muscle preservation procedure and the C6-nonpreservation procedure. The C6-preservation technique was not superior to the C6-nonpreservation technique in preventing postoperative axial neck pain.

\section{References}

1. Chiba K, Ogawa Y, Ishii K, Takaishi H, Nakamura M, Maruiwa H, et al: Long-term results of expansive open-door laminoplasty for cervical myelopathy-average 14-year follow-up study. Spine (Phila Pa 1976) 31:2998-3005, 2006

2. Estlander AM, Takala EP, Verkasalo M: Assessment of depression in chronic musculoskeletal pain patients. Clin J Pain 11:194-200, 1995

3. Fujimori T, Le H, Ziewacz JE, Chou D, Mummaneni PV: Is there a difference in range of motion, neck pain, and outcomes in patients with ossification of posterior longitudinal ligament versus those with cervical spondylosis, treated with plated laminoplasty? Neurosurg Focus 35(1):E9, 2013

4. Highsmith JM, Dhall SS, Haid RW Jr, Rodts GE Jr, Mummaneni PV: Treatment of cervical stenotic myelopathy: a cost and outcome comparison of laminoplasty versus laminectomy and lateral mass fusion. J Neurosurg Spine 14:619-625, 2011

5. Hirabayashi K, Miyakawa J, Satomi K, Maruyama T, Wakano K: Operative results and postoperative progression of ossification among patients with ossification of cervical posterior longitudinal ligament. Spine (Phila Pa 1976) 6:354-364, 1981

6. Hosono N, Sakaura H, Mukai Y, Fujii R, Yoshikawa H: C3-6 laminoplasty takes over C3-7 laminoplasty with significantly lower incidence of axial neck pain. Eur Spine J 15:1375 1379,2006

7. Hosono N, Sakaura H, Mukai Y, Yoshikawa H: The source of axial pain after cervical laminoplasty-C7 is more crucial than deep extensor muscles. Spine (Phila Pa 1976) 32:29852988, 2007

8. Hosono N, Yonenobu K, Ono K: Neck and shoulder pain after laminoplasty. A noticeable complication. Spine (Phila Pa 1976) 21:1969-1973, 1996

9. Itoh T, Tsuji H: Technical improvements and results of laminoplasty for compressive myelopathy in the cervical spine. Spine (Phila Pa 1976) 10:729-736, 1985

10. Johnson G, Bogduk N, Nowitzke A, House D: Anatomy and actions of the trapezius muscle. Clin Biomech (Bristol, Avon) 9:44-50, 1994

11. Johnson GM, Zhang M, Jones DG: The fine connective tissue architecture of the human ligamentum nuchae. Spine (Phila Pa 1976) 25:5-9, 2000

12. Kawaguchi Y, Kanamori M, Ishihara H, Ohmori K, Nakamura H, Kimura T: Minimum 10-year followup af- ter en bloc cervical laminoplasty. Clin Orthop Relat Res (411):129-139, 2003

13. Kawaguchi Y, Matsui H, Ishihara H, Gejo R, Yoshino O: Axial symptoms after en bloc cervical laminoplasty. J Spinal Disord 12:392-395, 1999

14. Kotani Y, Abumi K, Ito M, Sudo H, Takahata M, Ohshima $\mathrm{S}$, et al: Minimum 2-year outcome of cervical laminoplasty with deep extensor muscle-preserving approach: impact on cervical spine function and quality of life. Eur Spine J 18:663-671, 2009

15. Mori E, Okada S, Ueta T, Itaru Y, Maeda T, Kawano O, et al: Spinous process-splitting open pedicle screw fusion provides favorable results in patients with low back discomfort and pain compared to conventional open pedicle screw fixation over 1 year after surgery. Eur Spine J 21:745-753, 2012

16. Ono A, Tonosaki Y, Yokoyama T, Aburakawa S, Takeuchi K, Numasawa T, et al: Surgical anatomy of the nuchal muscles in the posterior cervicothoracic junction: significance of the preservation of the C7 spinous process in cervical laminoplasty. Spine (Phila Pa 1976) 33:E349-E354, 2008

17. Otani K, Sato K, Yabuki S, Iwabuchi M, Kikuchi S: A segmental partial laminectomy for cervical spondylotic myelopathy: anatomical basis and clinical outcome in comparison with expansive open-door laminoplasty. Spine (Phila Pa 1976) 34:268-273, 2009

18. Ratliff JK, Cooper PR: Cervical laminoplasty: a critical review. J Neurosurg 98 (3 Suppl):230-238, 2003

19. Sakaura H, Hosono N, Mukai Y, Fujii R, Iwasaki M, Yoshikawa H: Persistent local pain after posterior spine surgery for thoracic lesions. J Spinal Disord Tech 20:226-228, 2007

20. Seichi A, Takeshita K, Ohishi I, Kawaguchi H, Akune T, Anamizu Y, et al: Long-term results of double-door laminoplasty for cervical stenotic myelopathy. Spine (Phila Pa 1976) 26:479-487, 2001

21. Shiraishi T: A new technique for exposure of the cervical spine laminae. Technical note. J Neurosurg 96 (1 Suppl):122-126, 2002

22. Sivaraman A, Bhadra AK, Altaf F, Singh A, Rai A, Casey AT, et al: Skip laminectomy and laminoplasty for cervical spondylotic myelopathy: a prospective study of clinical and radiologic outcomes. J Spinal Disord Tech 23:96-100, 2010

23. Yukawa Y, Kato F, Ito K, Horie Y, Hida T, Ito Z, et al: Laminoplasty and skip laminectomy for cervical compressive myelopathy: range of motion, postoperative neck pain, and surgical outcomes in a randomized prospective study. Spine (Phila Pa 1976) 32:1980-1985, 2007

24. Vernon H: The Neck Disability Index: state-of-the-art, 19912008. J Manipulative Physiol Ther 31:491-502, 2008

\section{Author Contributions}

Conception and design: Mori. Acquisition of data: Mori, Ueta, Yugué. Analysis and interpretation of data: Mori, Yugué. Drafting the article: Mori. Critically revising the article: all authors. Reviewed submitted version of manuscript: all authors. Approved the final version of the manuscript on behalf of all authors: Mori. Administrative/technical/material support: Shiba.

\section{Correspondence}

Eiji Mori, Department of Orthopaedic Surgery, Spinal Injuries Center, 550-4 Igisu, Iizuka, Fukuoka 820-8508, Japan. email: eijimori@orange.ocn.ne.jp. 